\title{
110 - Investigations of Risk, Resilience and Novel Therapeutics of Late Life Neuropsychiatric Disease
}

Converging data from multiple domains of preclinical and human studies has underscored the importance of focusing on genetic, synaptic and neural circuity as critical neurobiological mechanisms of late life neuropsychiatric disease. These complementary research approaches have been applied to identify novel molecular mechanisms that may represent early intervention targets, as well as promising new treatments. The symposium will include four speakers who span the range of studies in animal models, post-mortem brain tissue, molecular imaging and clinical trials combined with biomarkers. $\mathrm{Dr}$ Etienne Sibille will present his research on the procognitive, neurotrophic and neurogenic effects of novel compounds augmenting dendritic inhibition and restoring neuronal connectivity, which is affected in aging and in neurodegenerative disorders, such as Alzheimer's disease. Dr. Robert Sweet will present new GWAS and human postmortem findings regarding risk/resilience to psychosis in Alzheimer's Disease, with an emphasis on synaptic mechanisms of resilience. Dr Helen Lavretsky will present a study of the neuroimaging, genetic and epigenetic effects of memantine and escitalopram treatment in late life depression. The strategy of incorporating biological measures into a clinical trial is an important opportunity to understanding the neurobiological mechanisms. Dr Gwenn Smith will present multimodality molecular imaging data to understand the synaptic changes associated with Alzheimer's Disease pathology in late life depression and mild cognitive impairment. Consideration of interdisciplinary research approaches and applications to different neuropsychiatric conditions may have particular relevance to understanding the neurobiological mechanisms underlying neuropsychiatric symptoms as risk factors or complications of neurodegenerative disease.

Molecular imaging methods to visualize the neuropathology of Alzheimer's disease (AD) in vivo provide an unprecedented opportunity to understand the neuropsychiatric (NPS) and cognitive symptoms observed in early stage AD by testing hypotheses informed by human neuropathology and animal models. A fuller understanding of the neurobiology of early $A D$ and its clinical progression is essential to identify individuals at risk and to identify targets for prevention and treatment. Numerous neuroimaging studies have shown that beta-amyloid and tau is necessary but not sufficient to explain cognitive decline and that models to explain cognitive decline must also include measures associated with synaptic dysfunction (eg cerebral glucose metabolism or brain volumes). Human data and animal models support the further investigation of serotonin $(5-\mathrm{HT})$ degeneration. Relative to other molecular targets, there is stronger evidence for 5-HT loss in both cognitive deficits and neuropsychiatric symptoms (NPS) in Alzheimer's Disease. 5-HT compounds are the only agents with preclinical evidence of multiple therapeutic mechanisms relevant to prevention and symptomatic treatment: blockade of amyloid precursor protein processing or neuroprotection, synaptic plasticity and improvement in both cognitive deficits and NPS. Multi-radiotracer PET studies of beta-amyloid (A $\beta$ ), tau and 5-HT have been performed longitudinally in amnestic, multi-domain, $\mathrm{MCl}(\mathrm{aMCl}-\mathrm{MD})$ and cognitively normal elderly. Cortical and limbic 5-HT degeneration was a more powerful predictor of longitudinal memory decline than A $\beta$ or Tau. Elucidating the role of $5-\mathrm{HT}$, in relation to Tau and $\mathrm{A} \beta$ in cognitive decline in aMCI-MD will have fundamental implications for the design of prevention and intervention studies targeting $5-\mathrm{HT}$.

\section{Title: Brain inhibitory GABAergic function and cognitive deficits in depression and during aging: Mechanisms and therapeutic targeting}

Changes in the brain excitation inhibition balance characterize neurological disorders and aging. Using human post-mortem samples and genetic rodent models, combined with genomic and bioinformatics approaches, we have reported changes in GABAergic inhibitory neurons that target pyramidal cell dendrites in human depression and during aging, and associated these changes with cognitive and mood behaviors in rodent models. Using molecular modeling, medicinal chemistry, pharmacological approaches and rodent behavioral models, we now show that novel molecules that activate alpha5- 